\title{
A prototype pavilion in textile reinforced concrete: a tool for research and pedagogy
}

\author{
Patricia Guaita, Raffael Baur, Miguel Fernández Ruiz, David Fernández- \\ Ordóñez
}

patricia.guaita@epfl.ch, raffael.baur@epfl.ch, miguel.fernandezruiz@upm.es, david.fernandez-ordonez@epfl.ch

\begin{abstract}
This paper presents a work performed in the last years on a Textile Reinforced Concrete (TRC) Prototype Pavilion, raising questions about research on architecture and engineering within a pedagogical context. The construction of the pavilion explores how a hands-on approach builds up multi-layered knowledge and constitutes a common ground of communication on which architects and engineers meet. Through the act of construction, architects and engineers work together, generating new knowledge and experiencing how tacit knowledge is built-up and transmitted. It also allows for the processes of conception and manufacture to feed and to enrichen each other. This action of making knowledge ( $\tau \dot{\varepsilon} \chi v \eta$, techne) constructs a thought or a concept as a tangible physical entity, acting as a communication interface between the work and the mind, spanning over different disciplines.

The paper highlights the fact that the act of building is not only an intellectual and technical task. Within an education context, students experience the complete process of observation, analysis, conception, execution and testing. The direct investigation of materiality is thus essentially an invention and innovation process: an iterative cycle building up knowledge through observation on the making. Through their corporal experience, students identify and engage with the research work, leading to collective action and to individual responsibility. Such engagement opens up perspectives on architectural, engineering as well as social, economic and environmental questions for the 21 st century: sustainable and resilient construction, economy of means, adequacy of expression.
\end{abstract}

\section{A Pavilion as an instrument to develop and to exchange knowledge}

This contribution is based on a long experience of pedagogical research in developing transdisciplinary strategies for designing and building projects in full scale and real-world conditions. The format of building a prototype construction by students of architecture and engineering offers the opportunity to work at the intersection between research and practice, of teaching and learning, of engineering and architecture. Our professions become more and more specialized and technical and thus we have less and less contexts to have an exchange of knowledge and to advance together in the necessity and evolution of developing the built environment. 
Within this context, this paper presents the process of conception and construction of a Textile Reinforced Concrete (TRC) Prototype Pavilion designed and built as part of a research collaboration between a team of architects and engineers at Ecole Polytechnique Fédérale de Lausanne (EPFL, Switzerland) [1]. The project was originated as an investigation on the potential of TRC to update lightweight building techniques with concrete (as argamassa armada [2]), and has evolved into an exploration in the pedagogy of making through prefabrication [3] and tectonic innovation.

The TRC Prototype Pavilion (see Fig. 1) was constructed during two summers at EPFL Fribourg by a group of students and researchers both from architecture and civil engineering. However, it is the result of a series of prior explorations during several academic years. Through observation, drawing and testing, different elements were developed. It is thanks to this iterative process that the necessary knowledge and innovation on the process of construction (formwork, reinforcement, concrete casting, curing and erection) is generated and allows defining useful and efficient building elements to constitute a prototype pavilion.

The TRC prototype pavilion is not thought as a final product, with a plan to be designed, built and exploited. On the contrary, it remains open to transformation. The interest of its construction is learning in the process, improving and understanding the potential of TRC as a sustainable alternative to ordinary concrete construction. The pavilion is thus intended to be a first step towards an adaptable tectonic system, a construction that will be further developed the next years and that leads to a new lightweight building paradigm, forecasting a new and different conception and image of concrete construction.
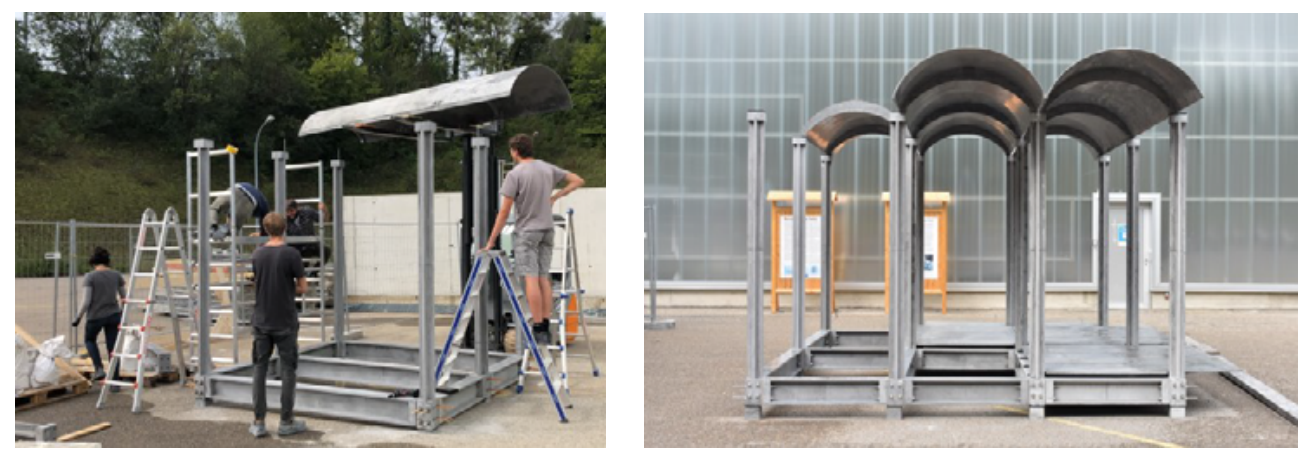

Fig. 1 TRC Prototype Pavilion: assembly and exposition of elements, EPFL Fribourg 2019 (photo Raffael Baur), EPFL Fribourg 2020 (photo Ana Carvalho).

\section{Materiality: lightness, structural performance and architectural language}

A major breakthrough in current construction approaches using cementitious-based materials (mostly concrete) can be identified in the use of non-corrosive reinforcement as for instance fabrics in carbon fibres, (see Fig 2). Such an approach removes the need to protect reinforcement from corrosion. As a consequence, reinforcement covers can be significantly reduced with respect to current values. Required centimetres for the covers in ordinary concrete construction become millimetres in TRC [1].

This leads to thinner and lighter structures, where the concrete matrix is used for static needs only and not for protection of reinforcement. In addition, the amounts of clinker required for the cementitious matrix can be dramatically reduced (no need for rebar passivation) reducing the total $\mathrm{CO}_{2}$ footprint of the construction. Other than sustainability aspects related to the environment, the possibility of building light-weight elements opens the door to self-construction by local communities. This allows for a socially-sustainable approach, allowing to build in high- and low-tech environments with a particular opening to the informal city. 
Despite the new potential offered by this material, a clear vision on how to implement it in construction is yet unclear. Probably, inspirations shall not come as an evolution from ordinary (massive) concrete construction, but from thin and lightweight elements, such as shells, Nervi's work in ferrocement and Filgueiras Lima (Lelé)'s work in argamassa armada [2]. Interestingly, the possibilities offered by a light material with a high potential to be prefabricated are also connected to steel construction and its approach to assemble and to connect pieces.

The construction of thin members with TRC allows for a rational manner to exploit the sustainable and durable qualities of TRC while affording spatial quality and allowing inhabitants of the informal city to participate in the production of their own shelter and habitat. The use of TRC allows to combine industrial processes (factory production) with local craftsmanship and labour. Such an approach allows for an efficient transfer of knowledge and technology to multiple environments, allowing also for its adaptation to local contexts. Another important aspect is the potential of TRC for recycling industrial waste products (as fly ash) and its potential capacity to be recycled in the future as for ordinary concrete construction. The use of precast elements in TRC, with convenient connections allows to create flexible and reusable structures in agreement with the concepts of circular economy.
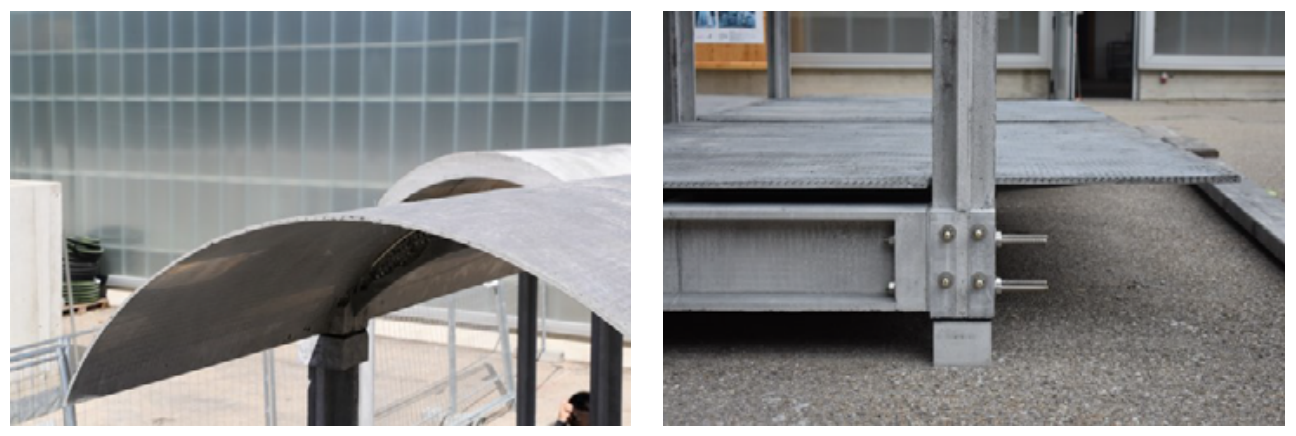

Fig. 2 TRC elements: shell 9mm thick, EPFL Fribourg 2019 (photo Graeg Eaves). Foundation beams, column, slab, EPFL Fribourg 2020 (photo Ana Carvalho).

\section{$3 \quad$ Atelier: tools and actions}

One of the hypothesis of the TRC research project is that craftsmanship constitutes a common ground on which architects and engineers meet. The practical, artisanal construction of a structural element and formwork system (i.e. a concrete reality which embodies a unity of architecture and engineering, of research and practical experience), does not demand for a division of analysis (calculation) and design but fosters a constructive understanding including the above mentioned environmental and social considerations.

Working in an atelier (studio) format allows the interaction of our hands and minds, which is an efficient manner to build up and exchange not only technical knowledge but also savoir faire and tacit knowledge between architects and engineers, teachers and students as well as between cultures of different continents.

In the process of life, neurologic structures emerge simultaneously with the development of skills and knowledge. Knowledge is a result of life, of its experiences and of the consciousness of individuals [4]. Every skill, also the most abstract ones, start from physical processes [5]. Understanding of techniques is established thanks to the application of an imagination on action. This process or "material consciousness" is the extension of thinking from the mind to the nervous system [6] and from the hand 
to the material world. Our hands are an interface between our brain and the real world, giving us the possibility to interact with ourselves, with materials and processes.

Thinking through action [7] allows understanding and solving situations and to develop an intuitive and tacit knowledge. In many cases, the work of the craftsman is done without a predefined theory of his own work, but by applying experience and intuition to precise contexts. Sharing experience, by dialogue and physical interaction, is thus the most efficient manner to transmit tacit knowledge [8]. Despite the fact that it is possible to distinguish between explicit and tacit knowledge, it is very hard to separate them in practice. Tacit knowledge is subjective and only by doing and through verbal exchange and physical discussion others can have access to it. Such a process generates a type of culture which can be identified as the addition of actions taken in response of specific contexts. One of the tasks of the architect and engineer is to transmit such a culture of tacit knowledge by means of different tools [9].

The distance between an architect or engineer and the process of making has become larger in the last decades as a result of intermediate actors [10]. Also, concepts and physical artefacts have become more and more separate. However, separating design and execution of a work separates the teaching and professional competences, leading to incomplete professional profiles in respect to the complexity of building.

In the atelier, learning is encouraged by the development of technical and spatial skills in reciprocal interaction, fostering a poetic understanding of construction, (Fig. 3). Supported on tools rooted in the core of the profession (drawing, models and physical prototypes), materials are transformed into spatial artefacts. Such actions promote intuition, sensitivity and knowledge transfer [11]. The work integrates all processes implied in construction: conceptual design, material innovation, fabrication and assembly. Such a physical approach is intuitive and allows students for a deeper understanding of building processes and to feel capable of modifying, transforming and developing our environment.
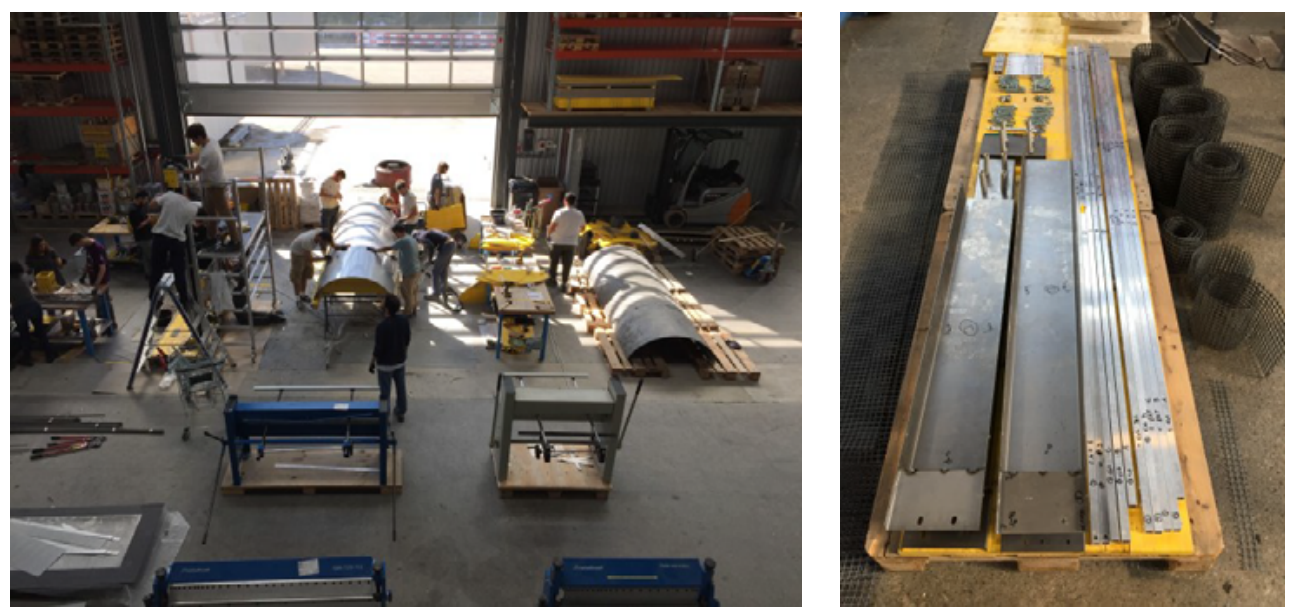

Fig. 3 Atelier Pop Up, EPFL Fribourg 2019, (photo Raffael Baur); prepared formwork and carbon net reinforcement of bean element, EPFL Fribourg 2019, (photo Raffael Baur).

\section{$4 \quad$ Interdisciplinary}

During the construction, students work directly with concrete. By designing, building and testing, they enter into a reciprocal relationship, such that construction becomes a projective activity. Everything is fabricated in the atelier, from sketch to prototype, drawing, formwork, reinforcing, casting, erecting and assembling. This way of working faces students to construction reality and its material qualities. 
Every generation of students inherits elements built by previous ones, thus a constructive language evolves. Their work is thus not isolated but belongs to a context and collaborative effort, to a culture of making. The construction of the pavilion shows that design and fabrication is a collective act. The time and effort involved in building with one's own hands reflect interactions with others.

Students need to understand and go through the complete process of analysis/observation, conception, execution and testing, (Fig. 4). This generates a deep learning impact in an interdisciplinary context. The gained experience and confidence of being actively involved in an innovation process encourages students to question and further develop any given task or reality in their coming careers. The interdisciplinary and collaborative nature of the process, which is necessary accounting for the complexity of the situation, can serve as model for any future activity.
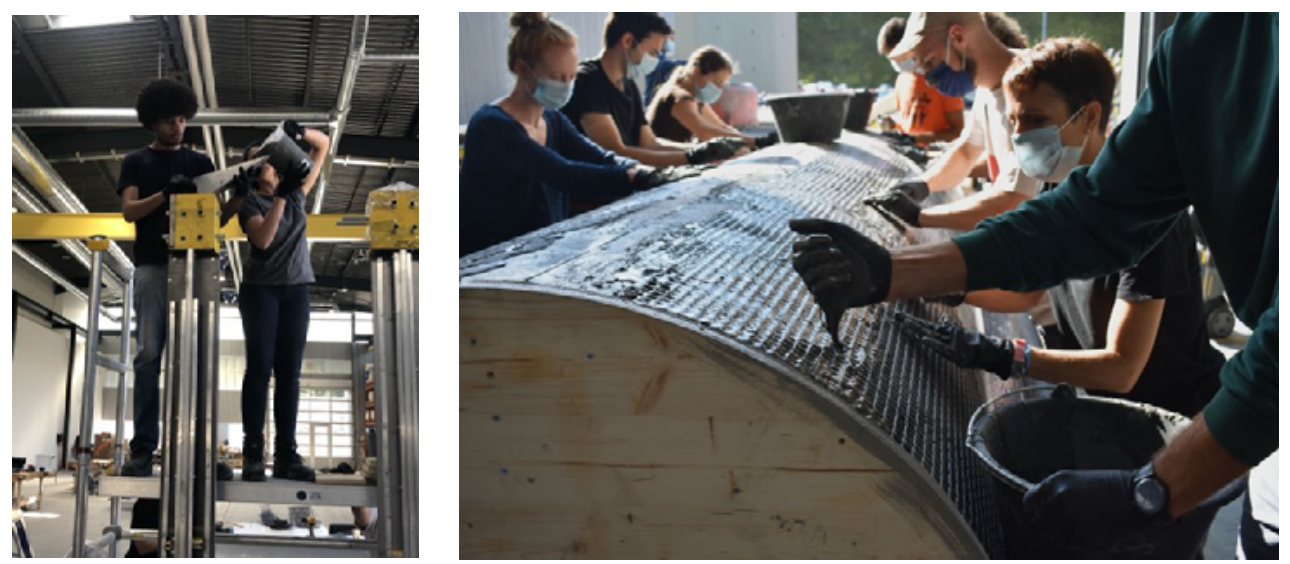

Fig. 4 Atelier Pop Up, experimental casting of the columns and shells. EPFL Fribourg 2019 (photo Sergio Ekerman), EPFL Fribourg 2020 (photo Ana Carvalho).

\section{$5 \quad$ Drawing as a cognitive tool}

Drawing constitutes a very powerful and critical tool of conceptual design. By articulating thought, it acts as a communication interface between the work and the mind [12] and between different disciplines; it is the most powerful language of communication in the working together between architects and engineers. The act of drawing is physically apprehended: the care in the drawing translates into a care in the making.

During this project on TRC, it is proposed to investigate analytical drawing methods capable of exploring structural and architectural concepts and solutions. Through analytical drawing, students enter into dialogue with the construction process in a direct manner. They get a sense for the adequacy of tools and refinement of solutions. This allows investigating the relation with scale and space, with tectonic articulation in relation to structural idea. An important contribution is also devoted to structural analysis, as the tool to make transparent the parameters and dependencies of the design process and to open the work to predict the structural response.

Drawing is a cognitive process where the dynamic relation between making and thinking is essential. The construction of points and lines on a piece of paper leads students to the notions of scale, size, proportion, transparency and composition, leading to build up their own tacit knowledge. The slow and tactile nature of the drawing process encourages the development and understanding of detail as a key 
moment of construction and as a mediator, relating a structure to the body and to the subject perceiving it.

Exploratory working drawings were done at different scales and incorporating calculation notes and a notion of sequence and time of the construction process, leading to generate a complete documentation of all the necessary information for the fabrication of a TRC element (meta-drawing, Fig. 5). This multi-scalar approach, opening and creating multiple relationships, overcomes typical difficulties of conventional presentations based on a linear sequence and acknowledges the potential of parallel and iterative processes in the conception and construction, allowing invention, experimentation and discoveries. These 'tactile' drawings allow the observing eye (mind) to survey (travel) from one detail to another while integrating all information into a mental construction.

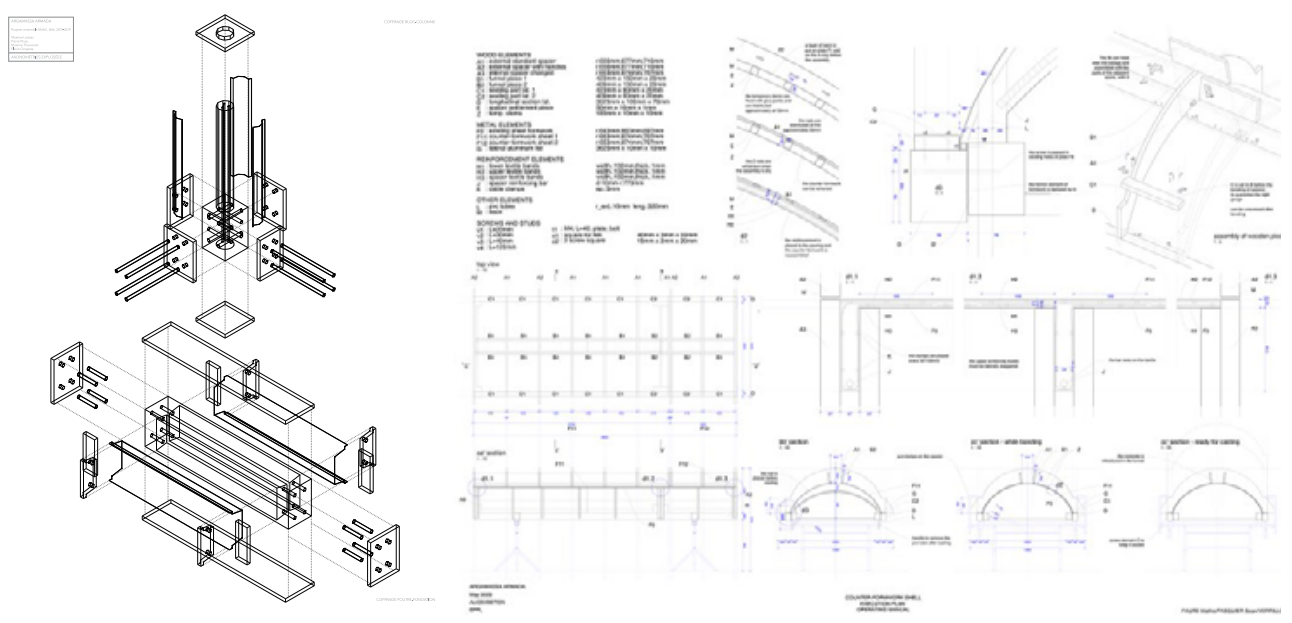

Fig. 5 Foundation beam, connection detail ENAC UE Argamassa Armada 2019 (drawing Flavio Gorgone) / Shell, ENAC UE Argamassa Armada 2020, (drawing Gianni Verrillo).

\section{Conclusion}

This paper reflected on the conception and fabrication process of an experimental pavilion in TRC, where the process of conception and manufacture feed each other to develop a thought or a concept into a concrete physical entity (Fig. 6).

It is shown that interactions between architects and engineers can occur naturally with the hand as inter-face between the ideas and the produced artefacts. In order to make a step forward in the process of building, it is important not only to share technical aspects, but mostly tacit knowledge. The latter, compiling multisensorial experiences, can only be transferred in an efficient manner by means of the making.

Working in an atelier format, with interdisciplinary teams composed of architects and engineers with different levels of experience and backgrounds, reveals to be a very powerful tool to address the challenges of tomorrow, opening a common ground for action and experience in between the body, nature and technology. 


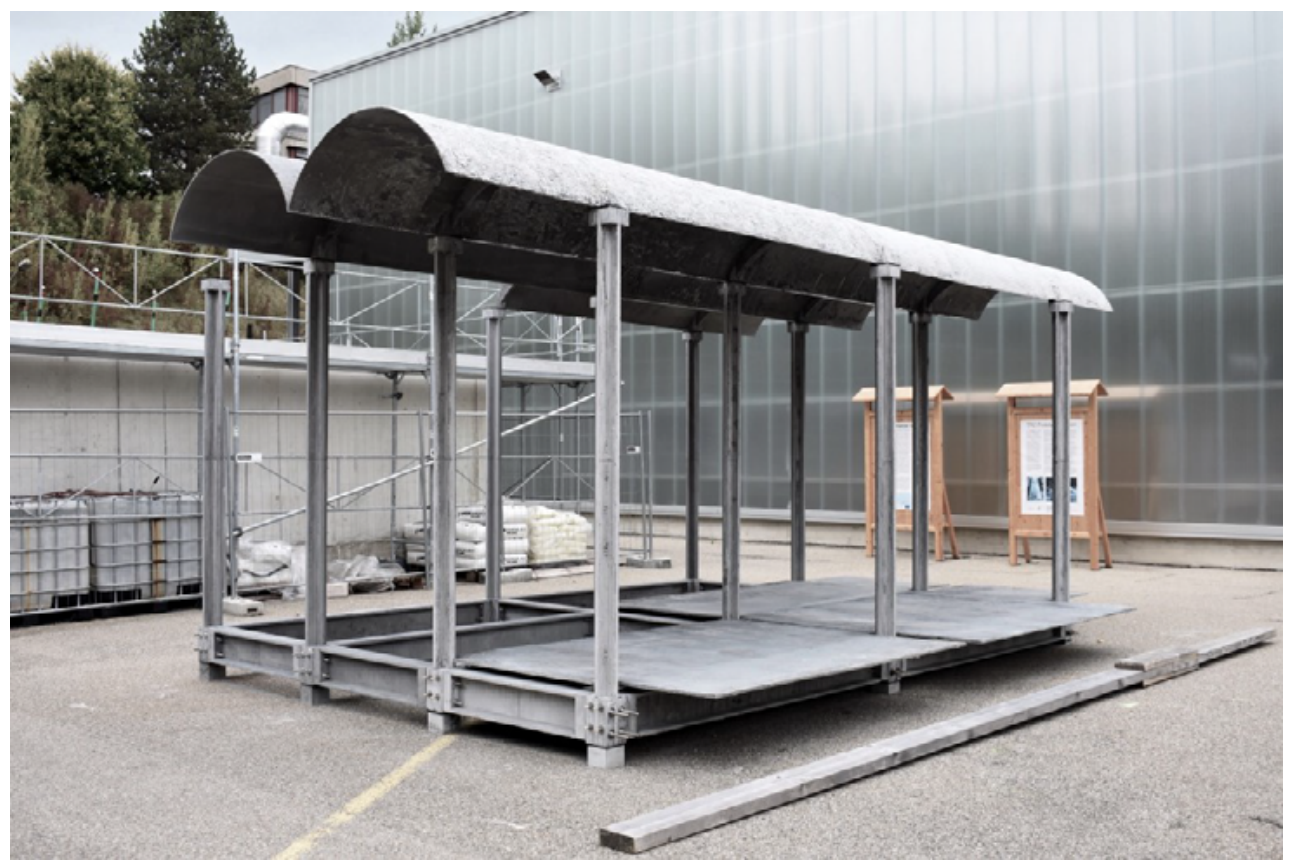

Fig. 6 TRC Prototype Pavilion, Atelier Pop Up, Structural Elements in Textile Reinforced Concrete, 2020 (photo Ana Carvalho).

\section{Acknowledgements}

This work has been partly funded by cemsuisse grant number 201801. The received support is greatly acknowledged by the authors.

The authors also sincerely acknowledge all the students that worked with us on this experience as well as the contribution of Prof. Sergio Ekerman from UFBA - Federal University of Bahia.

\section{References}

[1] Valeri, P., Guaita, P., Baur, R., Fernández Ruiz, M., Fernández-Ordóñez Hernández, D.C., Muttoni, A., 2020, "Textile Reinforced Concrete for sustainable structures: future perspectives and application to a prototype pavilion", Structural Concrete, Wiley, pp. 1-17 (https://doi.org/10.1002/suco.201900511)

[2] Lima, J. F., 2000. Joao Filgueiras Lima Lele: Brazilian Architects. Editorial Blau.

[3] Guaita, P., Valeri, P., Baur, P., Fernández Ruiz, M., 2018, "Pedagogy through construction: a dialogue between engineering and architecture by means of manual fabrication of Textile Reinforced Concrete elements", IV International Conference on Structural Engineering Education, ACHE, Madrid, Spain, 20-22 June, 11 p.

[4] Ingold, T., Afeissa, H.-S., \& Gosselin, H., 2017, Faire: Anthropologie, archéologie, art et architecture. Éditions Dehors.

[5] Sennett, R., 2010. Ce que sait la main: La culture de l'artisanat. Albin Michel.

[6] Wilson, F. R., \& Gavaldá, J., 2002. La mano: De cómo su uso configura el cerebro, el lenguage y la cultura humana. Tusquets Editores

[7] Schön, D. A., 2017. The reflective practitioner: How professionals think in action. Routledge. 
[8] Polanyi, M., 1966. The tacit dimension (Repr ed.). Gloucester, Mass.: Peter Smith.

[9] Sheil, B., 2005. Design through making: An introduction. Architectural Design, 75(4), 5-12.

[10] Turnbull, D. 1993. "The ad hoc collective work of building gothic cathedrals with templates, string, and geometry". Science, Technology, \& Human Values, 18(3), 315-340.

[11] Carvalho, J. J. D., \& Flórez-Flórez, J, 2014. "The meeting of knowledges: A project for the decolonization of universities in Latin America". Postcolonial Studies, 17(2), 122-139.

[12] Tversky, B., 1999. „What does drawing reveal about thinking?” In J. S. Gero and B. Tversky (eds), Visual and spatial reasoning in design. Sydney, Australia: Key Centre of Design Computing and Cognition, pp.93-101. 